\title{
Exchange Rate Forecasting Based on Combined Fuzzification Strategy and Advanced Optimization Algorithm
}

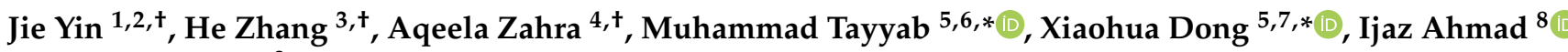 \\ and Nisar Ahmad ${ }^{9}$
}

check for updates

Citation: Yin, J.; Zhang, H.; Zahra, A.; Tayyab, M.; Dong, X.; Ahmad, I.; Ahmad, N. Exchange Rate Forecasting Based on Combined Fuzzification Strategy and Advanced Optimization Algorithm. Processes 2021, 9, 2204. https://doi.org/ $10.3390 /$ pr9122204

Academic Editors: Wen-Jer Chang, Hak Keung Lam and Yongming Li

Received: 15 November 2021

Accepted: 3 December 2021

Published: 7 December 2021

Publisher's Note: MDPI stays neutral with regard to jurisdictional claims in published maps and institutional affiliations.

Copyright: (c) 2021 by the authors. Licensee MDPI, Basel, Switzerland. This article is an open access article distributed under the terms and conditions of the Creative Commons Attribution (CC BY) license (https:/ / creativecommons.org/licenses/by/ $4.0 /)$.
1 College of Civil Engineering and Architecture, China Three Gorges University, Yichang 443002, China; yinjie2014cn2014@whu.edu.cn

2 School of Urban Design, Wuhan University, No. 8, Donghu South Road, Wuchang District, Wuhan 430072, China

3 School of Finance, Dongbei University of Finance and Economics, Dalian 116025, China; zhanghejb@126.com

4 School of Chemistry, Chemical Engineering and Life Sciences, Wuhan University of Technology, Wuhan 430070, China; zahra@whut.edu.cn

5 College of Hydraulic and Environmental Engineering, China Three Gorges University, Yichang 443002, China

6 College of Economics and Management, China Three Gorges University, Yichang 443002, China

7 Hubei Provincial Collaborative Innovation Center for Water Security, Wuhan 430070, China

8 Centre of Excellence in Water Resources Engineering, University of Engineering and Technology, Lahore 54890, Pakistan; ijaz.ahmad@cewre.edu.pk

9 School of Management, University of Science \& Technology (USTC), Hefei 230026, China; nisarwhu@mail.ustc.edu.cn

* Correspondence: mtayyab@ctgu.edu.cn (M.T.); xhdong@ctgu.edu.cn (X.D.); Tel.: +86-13227280848 (M.T.)

+ The authors Jie Yin, He Zhang, and Aqeela Zahra have contributed equally to this paper.

Abstract: Exchange rate forecasting is a crucial but challenging task due to the uncertainty and fuzziness of the associated data caused by complex influence factors. However, most traditional forecasting methods ignore the ambiguity of the data itself. Thus, in this paper, a novel fuzzy time series forecasting system based on a combined fuzzification strategy and an advanced optimization algorithm was proposed for use in exchange rate forecasting, and was proven to have an excellent ability to deal with the uncertainties and ambiguities in data. Concretely, the data "decomposition and ensemble" strategy was applied to carry out the data preprocessing process. The combined fuzzification strategy was used in the fuzzification of the observed data, and the advanced optimization algorithm was developed to determine the optimal parameters in the models. The analysis of this experiment verified the effectiveness of the proposed forecasting system, which will benefit future research and decision-making related to investments.

Keywords: exchange rate forecasting; fuzzy time series; combined fuzzification strategy; advanced optimization algorithm

\section{Introduction}

The financial market is a core component of economic operation. A financial time series is a time series of economic or financial indicators over time in the financial market. The analysis of financial time series is one of the crucial components in the analysis of the economic activities of an entire country. An exchange rate, a typical financial time series, is a ratio of a country's currency to other countries' currencies, which is one of the most commonly used financial instruments worldwide [1]. With the acceleration of economic globalization and financial integration, the issue of exchange rate has become a focus of concern for the international financial community. The process of changes in exchange rates is complicated and is closely related to a country's economic level, price level, inflation rate, interest rate, and government intervention. A country's exchange rate not only has a huge impact on the international economy and financial markets but also has 
an important impact on socioeconomic parameters and everyday life [2]. Therefore, in order to maintain sustained and stable economic growth, the correct prediction of exchange rates is imperative, as this can not only help the state and foreign trade enterprises strengthen management and avoid foreign exchange risks, but it is also a powerful supplement to the foreign exchange market theory [3].

However, exchange rates are greatly influenced by factors such as financial markets and national economies. Financial time series are always nonstationary, and high noise and always has long-term unpredictability. Changes in exchange rate time series changes are very complex, and the uncertainty and complexity of the exchange rate movement trend have a vital impact on exchange rate forecasting. Forecasting of exchange rates is a subject of wide interest to both academics and practitioners. In recent years, many studies regarding exchange rate forecasting have achieved relatively satisfactory results and have provided certain references for real financial markets.

The forecasting methods used with regard to exchange rates mainly include statistical models [4-6], artificial intelligence [7-10], and a combination of these two methods [3].

From the statistical point of view, some mathematical models for use in forecasting financial time series have been proposed. These models generally build statistical mathematical models based on existing observational data in order to find the mapping relationship between before and after time data in financial time series and use this mapping relationship to make short-term forecasts. In financial time series, the most commonly used statistical models include autoregressive integrated moving average (ARIMA), quantile regression (QR), and autoregressive conditional heteroskedasticity (ARCH). Balcilar [11] applied the QR model to forecast Bitcoin returns by Bitcoin trading volume. To improve the generalization of the QR model and reduce the probability of "overfitting", Bayer [12] proposed a penalized QR model based on the L1-norm and L2-norm in the elastic network and used the model to combine a series of value-at-risk forecasting sub models. The L1-norm and L2-norm can be used to effectively avoid the multicollinearity problem among the predicted values generated by sub models through coefficient compression and variable selection. The empirical results showed that the penalized quantile regression model outperforms the sub models and other benchmark models with higher forecasting accuracy. Iqbal et al. [13] used ARIMA models to forecast inflation rates and establish the appropriate model order based on the time series data. Zhang et al. [14] incorporated Taylor expansions based on tracking differentiators into ARIMA models, which were shown to effectively portray temporal correlation in time series. The empirical results based on future price forecasting showed that the proposed model has a significant improvement in forecasting accuracy relative to the benchmark model. You and Liu [15] applied the GARCH model in order to forecast the daily bilateral US exchange rates versus six currencies. The introduction of monthly monetary fundamental volatilities was shown to significantly improve the forecasting performance compared with the traditional time series volatility models.

However, these models often have restrictive statistical assumptions, such as normality, stability, etc., which have limitations in application. This is because the actual market data show that these assumptions have large deviations. Moreover, the financial time series is a complex, random, uncertain, and nonlinear system. Obviously, it is not accurate and objective to apply traditional statistical methods with many assumptions to forecast exchange rate fluctuations. Therefore, statistical models are limited in their applications to financial time series forecasting. At the same time, the volatility of exchange rates is affected by a variety of factors; it is difficult to guarantee the forecasting accuracy.

With the rapid development of computer, data science, and artificial intelligence technology, the artificial neural network and swarm intelligence algorithm has attracted increasing attention. It does not require statistical assumptions on the data and mostly uses metadata, which ensures the objectivity of the data. Moreover, because swarm intelligence algorithms study and simulate group activities in the biological world, with simple principles and good optimization effects, they are widely used in industrial control, 
natural sciences, and economics. Many studies have also tried to apply these theories to financial time series forecasting.

Wang et al. [16] proposed a stock index forecasting model based on wavelet denoising (WD) and the BP neural network (BPNN) and forecasted the monthly Shanghai Composite Index from January 1993 to October 2009. Tickno [17] proposed a Bayesian regularized artificial neural network (BRANN), which used the daily market prices of individual stocks and financial indicators as inputs to the forecasting model. The model was computationally efficient because it did not require data preprocessing, seasonal testing, and cycle analysis. He et al. [18] proposed a deep belief network ensemble model with empirical mode decomposition for use in the forecasting of exchange rates of USD against AUD, CAD, $\mathrm{CHF}$, and EUR. The proposed model demonstrated superior performance compared to that of the ARMA-GARCH model. Henríquez and Kristjanpoller [3] combined the independent component analysis and neural network models (ICA-NN) to forecast the exchange rate volatilities of both RMB/USD and RMB/EUR. The results indicated that compared with standard GARCH volatility models, the proposed forecasting model can better capture the RMB internationalization effect.

However, the highly complex and strongly nonlinear nature of exchange rates increases the difficulty of forecasting with time series. Traditional time series analysis assumes that the observed value is generated by the actual value and random error, that is, the uncertainty of the data is completely described by randomness, but the ambiguity of the sample data itself is ignored. Due to the fact that financial time series, in particular exchange rate time series, are characterized by uncertainty and randomness, Zadeh proposed a fuzzy set to provide a new research perspective with regard to financial time series. A fuzzy time series has the superior ability to deal with the uncertainties and ambiguities inherent in processing data collection. Compared with the artificial intelligent model and traditional statistical methods, fuzzy time series do not require a large number of samples for training, nor do they need a large number of hypothesis tests. Therefore, an increasing number of scholars have applied fuzzy time series for use in forecasting actual problems such as stocks and environment. An exchange rate is the result of a combination of factors, and the associated data are ambiguous. Thus, a novel fuzzy time series forecasting system based on the hesitant fuzzy sets (HFSs) and improved chaotic electromagnetic field optimization (ICEFO) algorithm was developed in this study for use in exchange rate forecasting. Concretely, the complete ensemble empirical mode decomposition with adaptive noise (CEEMDAN) was applied to weaken the effect of noise in the forecasting process. Then, the hesitant fuzzy sets with multiple fuzzification methods were developed to fuzzify the observed values. Otherwise, the ICEFO algorithm was applied to optimize the parameters, namely the weights, to aggregate the hesitant fuzzy sets. Accordingly, the main contributions and motivations of our study are summarized as follows:

(1) A novel fuzzy time series based on the hesitant fuzzy sets and advanced optimization algorithm was developed for use in exchange rate forecasting. The experiments verified the superior performance of the proposed forecasting system compared with competitive models.

(2) A fuzzy time series was applied in the forecasting system to deal with uncertain and fuzzy exchange rate data caused by complex influence factors.

(3) Hesitant fuzzy sets were developed to establish the multiple membership grade obtained by different fuzzification methods to fuzzify time series data.

(4) An improved chaotic electromagnetic field optimization algorithm was developed to determine the best aggregate weights for the hesitant fuzzy sets. Chaotic maps, a novel way to create electromagnetic particles, were introduced into the electromagnetic field optimization algorithm to improve the exploitation, convergence speed, and global optimization ability.

The rest of the paper is organized as follows: Section 2 introduces the structure of the forecasting system; Section 3 analyzes the experimental results; Section 4 discusses 
the statistical test results and practical applications; Section 5 provides the conclusion of the study.

\section{Design of the Forecasting System}

In our research, a novel hybrid forecasting system based on the HFSs and ICEFO algorithm was designed for use in exchange rate forecasting.

\subsection{Data Preprocessing Technique}

The CEEMDAN method was applied to preprocess the original time series of the exchange rate. The CEEMDAN method adaptively adds white noise to effectively solve the mode mixing phenomenon of empirical mode decomposition (EMD) and overcome the loss of completeness and reconstruction error of ensemble empirical mode decomposition (EEMD) after adding white noise [19].

EMD uses the process of "sieving" to decompose signals. For any given signal, $X(t)$, the cubic spline curve is used to connect all the maximum points to obtain the upper envelope and to connect all the minimum points to obtain the lower envelope. The difference between the data, $X(t)$, and the mean value of the upper and lower envelopes, $m_{1}$, is recorded as $h_{1}=X(t)-m_{1} . h_{1}$ is set as the new $X(t)$ and the above steps are repeated until $h_{1}$ meets the IMF conditions. The eigenmode components of the first stage are filtered out from the given signal.

The specific description of the algorithm is as follows.

(1) A standard normal distribution of white noise of a different amplitude, $\omega^{i}(n)$, is added to the given target signal; $x(n), \omega^{i}(n) \sim N(0,1)$ is the Gaussian white noise. The signal sequence of the $i$ th experiment is constructed:

$$
x^{i}(n)=x(n)+\gamma \omega^{i}(n)(i=1,2, \ldots, I)
$$

(2) In the first stage, EMD is applied to decompose the target signal to obtain the first modal component:

$$
\operatorname{IMF} F_{1}(n)=\frac{1}{I} \sum_{i=1}^{I} I M F_{1}^{i}(n)
$$

In the first stage, the residual signal is expressed as

$$
r_{1}(n)=x(n)-I M F_{1}(n)
$$

(3) $E_{k}(\cdot)$ is the $k$-th IMF modal component of a given signal obtained by EMD. The sequence $r_{1}(n)+\gamma_{1} E_{1}\left(\omega^{i}(n)\right)$ is decomposed to obtain the IMF component of the second stage:

$$
\operatorname{IMF}_{2}(n)=\frac{1}{I} \sum_{i=1}^{I} E_{1}\left\{r_{1}(n)+\gamma_{1} E_{1}\left(\omega^{i}(n)\right)\right\}
$$

The second residual signal is

$$
r_{2}(n)=r_{1}(n)-I M F_{2}(n)
$$

(4) Therefore, the $k$-th residual component is given as

$$
r_{k}(n)=r_{k-1}(n)-I M F_{k}(n)
$$

Then, the $k+1$-th IMF component is given as

$$
I M F_{k+1}(n)=\frac{1}{I} \sum_{i=1}^{I} E_{1}\left\{r_{k}(n)+\gamma_{k} E_{k}\left(\omega^{i}(n)\right)\right\}
$$


(5) Step (4) is repeated until the residual component cannot meet the EMD decomposition condition or the iteration comes to an end. Then, the target signal, $x(n)$, is decomposed into

$$
x(n)=\sum_{k=1}^{K} I M F_{k}(n)+R(n)
$$

$R(n)$ is the final residual component, also known as the residual component.

\subsection{Fuzzy Processing}

In the fuzzy processing section of this study, some key concepts need to be introduced, including fuzzy set, hesitant fuzzy set, and fuzzy times series.

Definition 1. Fuzzy set (FS).

Suppose that a given mapping $\mu_{A}: U \rightarrow[0,1]$ on the universe of discourse such that $x \in U \rightarrow \mu_{A}(x) \in[0,1]$, then it is said that a fuzzy subset of the universe of discourse is determined, referred to as a fuzzy set. $\mu_{A}$ is the membership function. Generally, the fuzzy set is expressed as $A=f_{A}\left(x_{1}\right) / x_{1}+f_{A}\left(x_{2}\right) / x_{2}+\cdots+f_{A}\left(x_{n}\right) / x_{n} ;\left\{f_{A}\left(x_{i}\right)\right\}(i=1,2, \ldots, n)$ is the membership function [20].

Definition 2. Fuzzy time series.

A new dynamic process composed of fuzzy sets is called a fuzzy time series. Let $Y(t)(t=0,1,2, \ldots)$ be a subset of the real number set, $f_{i}(t)(i=1,2,3, \ldots)$ is the fuzzy set defined on the universe of discourse, and $F(t)=\left\{f_{1}(t), f_{2}(t), \cdots\right\}$ is the fuzzy time series defined on $Y(t)[21]$.

Definition 3. Hesitant fuzzy set (HFS).

A hesitant fuzzy set was defined by Torra (2010) to consist of a set of elements and corresponding degrees of membership. Based on the function $h$, the hesitant fuzzy set $(H)$ is defined by $X$, which is a subset of $[0,1][22]$.

$$
H=\{\langle x, h(x)\rangle \mid x \in X\}
$$

For example, if set $X=\left\{x_{1}, x_{2}, x_{3}\right\}$ is the reference set and $h_{H}\left(x_{1}\right)=\{0.2,0.3,0.5\}$, $h_{H}\left(x_{2}\right)=\{0.2,0.4,0.6\}$, and $h_{H}\left(x_{3}\right)=\{0.2,0.5\}$ are the possible memberships, then the HFS is expressed as $H=\left\{\left\langle x_{1},\{0.2,0.3,0.5\}\right\rangle,<x_{2},\{0.2,0.4,0.6\}\right\rangle,\left\{\left\langle x_{3},\{0.2,0.5\}\right\rangle\right\}$. The three main operation rules between two HFSs are as follows.

$$
\begin{gathered}
h_{1} \cup h_{2}=\left\{\gamma_{1} \vee \gamma_{2} \mid \gamma_{1} \in h_{1}, \gamma_{2} \in h_{2}\right\} \\
h_{1} \cap h_{2}=\left\{\gamma_{1} \wedge \gamma_{2} \mid \gamma_{1} \in h_{1}, \gamma_{2} \in h_{2}\right\} \\
h_{1}^{c}=\left\{1-\gamma \mid \gamma \in h_{1}\right\}
\end{gathered}
$$

Here, $\wedge$ and $\vee$ are min and max operators.

Furthermore, in order to establish a fuzzy set that can be used for further calculations, the order-weighted average (OWA) operator is generally used to integrate hesitant fuzzy elements.

Set $R^{n} \rightarrow R$ is the function with $n$ variables, $W=\left(w_{1}, w_{2}, \cdots, w_{n}\right)$ is the related weight matrix, and $\sum_{j=1}^{n} w_{j}=1, w_{j} \geq 0, j=1,2, \ldots, n . \operatorname{OWA}\left(a_{1}, a_{2}, \cdots, a_{n}\right)=\sum_{j=1}^{n} w_{j} b_{j}$, $b_{j}$ is the $n$-th number in descending order of $a_{1}, a_{2}, \cdots, a_{n}$. Then, OWA is named the $n$ dimensional order-weighted average operator. OWA satisfies the condition that $\min \left(\left\{a_{1}, a_{2}, \cdots, a_{n}\right\}\right) \leq \boldsymbol{O W A}\left(\left\{a_{1}, a_{2}, \cdots, a_{n}\right\}\right) \leq \max \left(\left\{a_{1}, a_{2}, \cdots, a_{n}\right\}\right), \forall a_{1}, a_{2}, \cdots, a_{n} \in$ $[0,1]$. 


\subsection{Forecasting Process}

A fuzzy time series with a hesitant fuzzy set was applied to the forecasting process $[23,24]$. The detailed steps of the forecasting process as follows.

Step 1. The original data is preprocessed based on the decomposition and ensemble strategy. Then, the universe of discourse is determined: $U=\left[X_{\min }-\sigma, X_{\max }+\sigma\right]$. Here, $X_{\min }$ and $X_{\max }$ are the minimum and maximum of the data after preprocessing, respectively, and $\sigma$ is the variance of the data.

Step 2. The fuzzification of data mainly depends on the universe of discourse partition and the membership function. The combined fuzzified method with the triangular membership function is applied to obtain the intervals and membership degree. The combined fuzzified methods include equal width (EW) [25], information granularity (IG) [26], and kernel fuzzy c-means clustering (KFCM) [27]. The triangular membership function is given as follows:

$$
f_{A}(x, a, r, b)= \begin{cases}\frac{x-a}{r-a} & a \leq x<r \\ 1 & x=r \\ \frac{x-b}{r-b} & r<x<b \\ 0 & \text { others }\end{cases}
$$

In this study, $a, r, b$ are the left end, midpoint, and right end of the intervals, respectively. Then, the membership degree of the observations belongs to different subintervals under three universe of discourse partition methods.

Step 3. The hesitant fuzzy set is constructed and the membership degrees of a certain observation, $x$, are aggregated using three different methods. The hesitant fuzzy sets are constructed as

$$
H=\{\langle x, h(x)\rangle \mid x \in X\}
$$

The hesitant fuzzy sets $H=\left\{h_{1}, h_{2}, \cdots, h_{N}\right\}$ are aggregated by the order-weighted average (OWA) operator to obtain the fuzzy set.

$$
\begin{gathered}
H_{A}=\left\{\left\langle x, \boldsymbol{O W A}\left(h_{H}(x)\right)\right\rangle \mid x \in X\right\} \\
\boldsymbol{O W A}\left(h_{H}(x)\right)=\sum_{j=1}^{n} w_{j} h_{H}(x)_{j}
\end{gathered}
$$

The ICEFO algorithm is used to determine the best weight $w$, in Equation $(16)[28,29]$. $\mathrm{EFO}$ is a population-based heuristic global optimization algorithm. Each solution vector (electromagnetic particle) is represented by a group of electromagnets [28]. The detailed steps can be summarized as follows:

1. Generate the initial population $\mathbf{P O P}=\left[\boldsymbol{P O P}_{1}, \mathbf{P O P}_{2}, \cdots \boldsymbol{P O P}_{N}\right]$, namely the electromagnetic particles (EMPs), $\mathbf{P O P}_{i}=\left[p_{i}^{1}, p_{i}^{2}, \cdots, p_{i}^{d}, \cdots p_{i}^{D}\right] ; N$ is the population size and $D$ is the dimension of $\boldsymbol{P O P}$. Then, calculate the fitness value, $f\left(\boldsymbol{P O P}_{\boldsymbol{i}}\right)$, and sort the particles according to $f\left(\boldsymbol{P O P}_{\boldsymbol{i}}\right)$ values.

2. Divide the sorted particles into three groups:

$$
\begin{aligned}
& P \_ \text {area }=\left(1, n \times P \_ \text {field }\right) \\
& N \_ \text {area }=\left(n \times\left(1-N \_ \text {field }\right), n\right) \\
& K \_ \text {area }=\left(n \times P \_ \text {field }, n \times\left(1-N \_ \text {field }\right)\right)
\end{aligned}
$$

where $P_{-}$field is the positive field ratio and $N_{-}$field is the negative field ratio. The first group is a positive electromagnetic field, which is composed of electromagnetic particles with the best fitness value, and these particles are positive; the second group is a negative electromagnetic field, which is composed of electromagnetic particles with the worst fitness value, and these particles are negative; the third group is a neutral magnetic field, which consists of the remaining electromagnetic particles, and the negative polarity of these particles is almost zero. 
3. Generate a random number, $r_{1} \in(0,1)$, compare it with the updated probability Ps_rate, and determine the updated method for each dimension of the electromagnetic particle. If $r_{1}<P P_{-}$rate, the electromagnet is obtained directly from the positive electric field of the same dimension, that is

$$
E M P_{j}^{n e w}=E M P_{j}^{P}
$$

where $d(d=1,2, \ldots, D)$ is the dimension of the particle; $P$ is a random integer of the positive electromagnetic field and $P \in P \_$area; $E M P_{j}^{P}$ is the $j$ th electromagnet on an electromagnetic particle randomly selected from the positive electromagnetic field. If $r_{1} \geq P$ s_rate, an electromagnet is randomly selected from the same dimension of three electromagnetic fields with different polarities and brought into the following updated equation to change the position.

$$
E M P_{j}^{n e w}=E M P_{j}^{n e u t r a l}+(\varphi \times r) \times\left(E M P_{j}^{\text {positive }}-E M P_{j}^{\text {neutral }}\right)-r \times\left(E M P_{j}^{\text {negative }}-E M P_{j}^{\text {neutral }}\right)
$$

where $\varphi=(1+\sqrt{5}) / 2$ is the golden ratio, and $\mathrm{r}$ is the random number between 0 and 1 called the force of the electromagnetic particle. $E M P_{j}^{\text {new }}$ is the new particle at the $j$ th dimension and $E M P_{j}^{\text {positive }}, E M P_{j}^{\text {neutral }}$, and $E M P_{j}^{\text {negative }}$ are the electromagnetic particles from three field groups.

4. In order to prevent the algorithm from falling into the local optimum and to maintain the diversity of the population, for some newly generated electromagnetic particles, only the electromagnet in a certain dimension is replaced by a randomly generated electromagnet. Generate a random number, $r_{2} \in(0,1)$, and compare it with the mutation probability $R \_$rate. If $r_{2}<R \_$rate, then one dimension of the new particle is mutated.

5. Calculate the fitness value of the new electromagnetic particle and compare it with the fitness value of the worst electromagnetic particle in the original population. Retain the better $N$ fitness and update the population.

6. According to the above process, the position of each particle is updated, and one iteration of the EFO algorithm is completed. Stop the iteration when the requirement is met.

The improved chaotic electromagnetic field optimization (ICEFO) algorithm is an improvement of the EFO algorithm. One of the key components to the EFO algorithm is the degree of chaos of the particles. The greater the degree of chaos, the stronger the search power of the algorithm. In this paper, chaotic processing was performed on the position of the initial electromagnetic particles, which disturbed the distribution of electromagnetic particles and increased the unpredictability of the initial state of the system. The chaos phenomenon refers to external complex behaviors due to internal randomness in a certain system, which manifests as uncertainty, unrepeatability, and unpredictability. Among many chaotic maps, the Logistic chaotic map is widely used because of its simple expression and good random performance. A disadvantage of the Logistic map is that the chaotic sequence is not uniformly distributed. Thus, in this paper, four chaotic maps (Logistic map, Chebyshev map, Circle map, and Iterative chaotic map) are introduced [29].

Another improvement to the EFO algorithm is the way in which new particles are generated. Four new particles generated by the four abovementioned chaotic maps are compared, and the optimal particles are selected. Therefore, Equation (19) can be rewritten as Equation (20).

$$
\begin{gathered}
E M P_{j}^{\text {new }}(k)= \\
\qquad M P_{j}^{\text {neutral }}(k)+(\varphi \times r(k)) \times\left(E M P_{j}^{\text {positive }}(k)-E M P_{j}^{\text {neutral }}(k)\right)- \\
r(k) \times\left(E M P_{j}^{\text {negative }}(k)-E M P_{j}^{\text {neutral }}(k)\right), k=1,2,3,4
\end{gathered}
$$

where $k$ denotes the number of chaotic maps and $r(k)$ denotes the force of the particle generated from the different chaotic maps. 
The EFO algorithm is improved with chaotic maps, and the method through which new EMPs are generated is called the improved chaotic electromagnetic field optimization (ICEFO) algorithm.

Based on the weight obtained by the ICEFO algorithm, the HFs are transformed into FS. According to the aggregated FS, the observations are fuzzified by the principle of the maximum membership degree.

Step 4. Establish the fuzzy logical relationship so that the fuzzy logical group is obtained. $\forall f_{i}(t) \in F(t), \exists f_{i}(t-1) \in F(t-1)$ and $R_{i j}(t, t-1)$, then $f_{j}(t)=f_{i}(t-1) \circ R_{i j}(t, t-1)$. "o" is the max-min operator, $F(t)$ is only affected by $F(t-1)$, expressed as $f_{i}(t-1) \rightarrow f_{j}(t)$. $R(t, t-1)$ is called the fuzzy logical relationship (FLR) between $F(t)$ and $F(t-1)$. Set $F(t-1)=A_{t-1}$ and $F(t)=A_{t}$; the relationship between $F(t)$ and $F(t-1)$ can be expressed as $A_{i} \rightarrow A_{j} . A_{i}$ is the left-hand side and $A_{j}$ is the right-hand side; the FLR at the same left-hand side can be grouped as the fuzzy logical relationship group (FLRG). For example, $\left\{A_{1} \rightarrow A_{2}, A_{1} \rightarrow A_{3}, A_{1} \rightarrow A_{3}\right\}$ can be grouped as $\left\{A_{1} \rightarrow A_{2}, A_{3}\right\}$.

Step 5. Calculate the forecasting values. The fuzzy transition weight matrix $\left(W \_s\right)$ is calculated based on the FLRG, and then, the centroid defuzzification method is applied to calculate the final forecasting results. The forecasting result at time $t(f(t))$ is

$$
\begin{gathered}
f(t)=\sum_{j} w s_{i j} \cdot m_{i} \\
m_{i}=m_{i}^{1} w_{i}^{1}+m_{i}^{2} w_{i}^{2}+m_{i}^{3} w_{i}^{3}
\end{gathered}
$$

where $w s_{i j}$ is the element of the standardized fuzzy transition weight matrix $\left(W \_s\right) ; m_{i}^{1}, m_{i}^{2}$, and $m_{i}^{3}$ are the midpoints of the $i$ th subinterval under three partition methods; $w_{i}^{1}, w_{i}^{2}$, and $w_{i}^{3}$ are the weights obtained by the ICEFO algorithm for the $i$ th subinterval under three partition methods.

\section{Experimental Results Analysis and Discussion}

In this section, the data, related processes, and results of the experiment are analyzed.

\subsection{Data Description}

In this study, both the USD/RMB and EURO/RMB exchange rates were subject to experimental analysis. The dataset regarding the monthly USD/RMB exchange rate covered the period from January 1994 to March 2020. The top 236 data were used to establish the model, and the rest of the data were used to test the performance of the forecasting system. The EURO/ RMB exchange rate sequence was analyzed from April 2002 to May 2020. The top 164 data were used to establish the model and the rest of the data were used to test the performance of the forecasting system. The statistical description of the different datasets is presented in Table 1. As shown in Figure 1, the time series fluctuate in their complexity and strong nonlinearity, which increases the difficulty of forecasting with time series. Thus, the accurate forecasting of exchange rates is challenging.

Table 1. Statistical description indicators of the exchange rate datasets.

\begin{tabular}{lllllllll}
\hline Statistical Indicators & Number & Maximum & Minimum & Median & Mean & IQR & Std. \\
\hline \multirow{4}{*}{ USD/RMB } & Total & 315 & 8.710 & 6.100 & 7.740 & 7.458 & 1.625 & 0.871 \\
& Training & 236 & 8.710 & 6.170 & 8.280 & 7.763 & 1.440 & 0.778 \\
& Testing & 78 & 7.090 & 6.100 & 6.600 & 6.546 & 0.700 & 0.326 \\
& Total & 218 & 11.080 & 6.630 & 8.435 & 8.761 & 2.040 & 1.168 \\
EURO/RMB & Training & 164 & 11.080 & 6.630 & 9.215 & 9.138 & 1.830 & 1.106 \\
& Testing & 54 & 7.980 & 7.020 & 7.695 & 7.619 & 0.400 & 0.231 \\
\hline
\end{tabular}



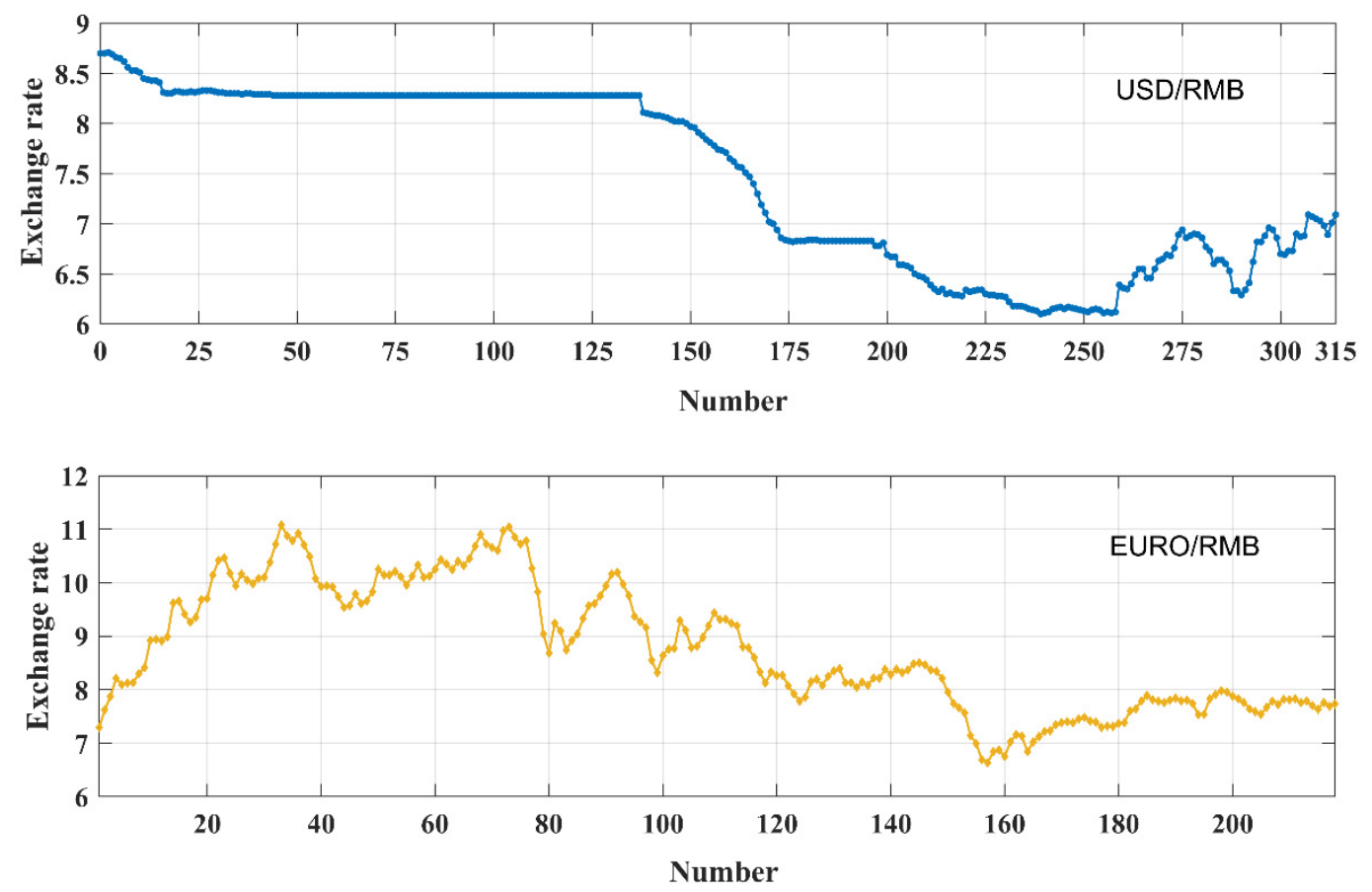

Figure 1. Data of the USD/RMB and EURO/RMB exchange rates.

\subsection{Evaluation Criteria}

To verify the forecasting performance of the proposed forecasting system for use in exchange rate datasets, some statistical indicators, including mean absolute percentage error (MAPE), root mean square error (RMSE), mean absolute error (MAE), index of agreement of forecasting results (IA), and the variance of the forecasting error (VAR), were used to evaluate the forecasting accuracy and stability of the different forecasting methods [30]. All of the indicators were based on the forecasting errors; MAPE, RMSE, $\mathrm{MAE}$, and IA were used to measure the forecasting accuracy, while VAR was used to measure the forecasting stability. The calculation formulae of the indicators are presented in Table 2.

Table 2. Calculation formulae of the evaluation formulae.

\begin{tabular}{ll}
\hline Indicators & Formula \\
\hline MAE & MAE $=\frac{1}{N} \sum_{i=1}^{N}\left|y_{i}-\hat{y}_{i}\right|$ \\
RMSE & RMSE $=\sqrt{\frac{1}{N} \times \sum_{i=1}^{N}\left(y_{i}-\hat{y}_{i}\right)^{2}}$ \\
MAPE & MAPE $=\frac{1}{N} \sum_{i=1}^{N}\left|\frac{y_{i}-\hat{y}_{i} \mid}{y_{i}}\right| \times 100 \%$ \\
IA & IA $=1-\sum_{i=1}^{N}\left(y_{i}-\hat{y}_{i}\right)^{2} / \sum_{i=1}^{N}\left(\left|y_{i}-\bar{y}\right|+\left|y_{i}+\bar{y}\right|\right)^{2}$ \\
VAR & Var $=E(\hat{e}-E(\hat{e}))^{2}$ \\
\hline
\end{tabular}

\subsection{Experimental Results Analysis}

In our experiment's design, some commonly used forecasting methods, including two statistical models (ARIMA and DES), two artificial intelligent models (BP and ELM), and an SVR model, were selected for comparison.

As presented in Table 3, under all of the five indicators, the proposed forecasting system outperformed the other comparison models with regard to the two datasets. Regarding forecasting accuracy, the MAPE values of the proposed forecasting system and the other five methods were $0.7451 \%, 0.9971 \%, 1.2623 \%, 0.8045 \%, 0.8484 \%$, and $0.9394 \%$, respectively, 
for USD/RMB exchange rate forecasting. For the forecasting stability, the variance in the forecasting errors of these methods was $0.0047,0.0083,0.0121,0.0060,0.0066$, and 0.0083 , respectively. Except for the proposed forecasting system, the SVR model possessed the best performance for both USD/RMB and EURO/RMB exchange rate forecasting. Otherwise, the statistical models were all superior to the artificial intelligence methods with regard to forecasting the two exchange rates. As is widely known, the performance of artificial intelligence can be significantly affected by the size of the sample used for training. Thus, under the restriction of the sample size, the statistical models may display better performance than artificial intelligence. Take the USD/RMB exchange rate, for example; the forecasting results and errors are depicted in Figure 2. The trend shown with regard to the forecasting value is in accordance with the actual value in general. All of the errors are between -0.6 and 0.4 , while the error of the proposed forecasting system is between -0.2 and 0.2 .

Table 3. Evaluation indicators of the forecasting results obtained by different methods.

\begin{tabular}{|c|c|c|c|c|c|c|c|}
\hline \multirow{2}{*}{$\begin{array}{l}\text { Exchange } \\
\text { Rate }\end{array}$} & \multirow[b]{2}{*}{ Indicators } & \multirow[b]{2}{*}{ Proposed } & \multicolumn{2}{|c|}{ Artificial Intelligence } & \multirow[b]{2}{*}{ SVR } & \multicolumn{2}{|c|}{ Statistical Models } \\
\hline & & & BP & ELM & & $\begin{array}{l}\text { ARIMA } \\
(1,1,3)\end{array}$ & DES \\
\hline \multirow{5}{*}{$\begin{array}{l}\text { USD/ } \\
\text { RMB }\end{array}$} & $\begin{array}{l}\text { MAPE } \\
(\%)\end{array}$ & 0.7451 & 0.9971 & 1.2623 & 0.8045 & 0.8484 & 0.9394 \\
\hline & RMSE & 0.0720 & 0.0907 & 0.1203 & 0.0819 & 0.0807 & 0.0904 \\
\hline & MAE & 0.0494 & 0.0663 & 0.0845 & 0.0536 & 0.0565 & 0.0624 \\
\hline & IA & 0.9873 & 0.9801 & 0.9626 & 0.9835 & 0.9848 & 0.9811 \\
\hline & VAR & 0.0047 & 0.0083 & 0.0121 & 0.0060 & 0.0066 & 0.0083 \\
\hline \multirow[b]{2}{*}{$\begin{array}{l}\text { Exchange } \\
\text { Rate }\end{array}$} & \multirow[b]{2}{*}{ Indicators } & \multirow[b]{2}{*}{ Proposed } & \multicolumn{2}{|c|}{ Artificial Intelligence } & \multirow[b]{2}{*}{ SVR } & \multicolumn{2}{|c|}{ Statistical Models } \\
\hline & & & BP & ELM & & $\begin{array}{l}\text { ARIMA } \\
(5,1,3)\end{array}$ & DES \\
\hline \multirow{5}{*}{ EURO/RMB } & $\begin{array}{l}\text { MAPE } \\
(\%)\end{array}$ & 0.8536 & 1.8282 & 1.5294 & 1.1451 & 1.1455 & 1.2083 \\
\hline & RMSE & 0.0865 & 0.1734 & 0.1500 & 0.1134 & 0.1026 & 0.1226 \\
\hline & BMAE & 0.0648 & 0.1380 & 0.1166 & 0.0871 & 0.0870 & 0.0917 \\
\hline & IA & 0.9679 & 0.8974 & 0.9077 & 0.9438 & 0.9540 & 0.9394 \\
\hline & VAR & 0.0057 & 0.0179 & 0.0170 & 0.0080 & 0.0104 & 0.0153 \\
\hline
\end{tabular}

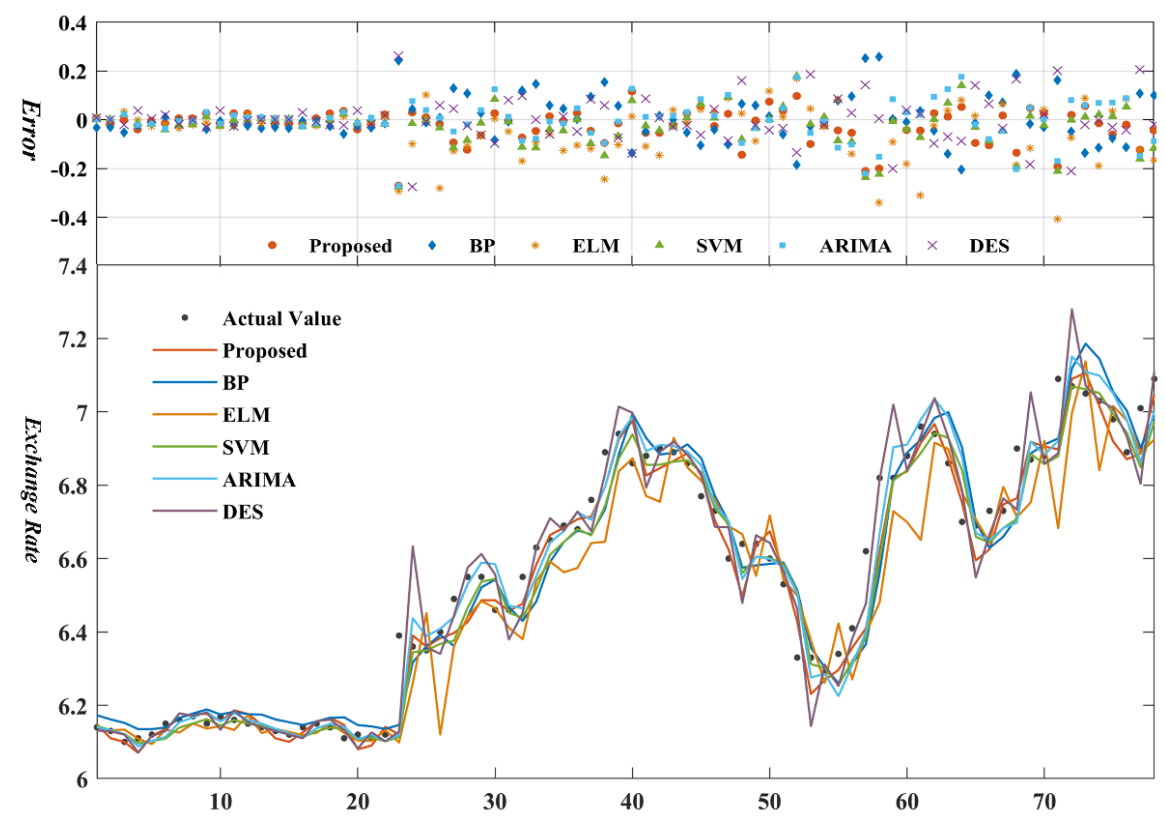

Figure 2. Forecasting results an d error of the USD/RMB exchange rate. 


\section{Discussion}

\subsection{Statistical Test}

In the previous section, several indicators were used to evaluate the forecasting performance. However, an individual indicator cannot verify the difference in performance between the proposed forecasting system and the compared models. Thus, a statistical test, namely the Diebold-Mariano (DM) test, was applied to test the forecasting accuracy difference between the two competitive models [31].

The test results are presented in Table 4. Compared with the critical value under different significant levels, most of the DM statistics are higher than $Z_{0.01 / 2}=2.58$, and all of the values are higher than $Z_{0.1 / 2}=1.64$. Thus, under $1 \%$ significance level, the performance of the proposed forecasting system is significantly different from the competitive models in most cases, and under $10 \%$ significance level, the same conclusion can be found in all cases. By combining these results with the evaluation results, it can be concluded that the proposed forecasting system is evidently superior to the competitive models.

Table 4. DM test results of the proposed system and competitive models.

\begin{tabular}{lllllll}
\hline & BP & ELM & SVM & ARIMA & GM & DES \\
\hline USD/RMB & $3.2093^{* * *}$ & $3.6755^{* * *}$ & $2.4026^{* *}$ & $1.7979^{*}$ & $8.8995^{* * *}$ & $2.0312^{* *}$ \\
EURO/RMB & $5.0426^{* * *}$ & $3.3231^{* * *}$ & $3.3457^{* * *}$ & $1.9976^{* *}$ & $5.0765^{* * *}$ & $2.7339^{* * *}$ \\
\hline N & &
\end{tabular}

Note: ${ }^{* * *} 1 \%$ significance level, ${ }^{* *} 5 \%$ significance level, ${ }^{*} 10 \%$ significance level.

\subsection{The Practical Application of the Exchange Rate Forecasting}

In 2016, RMB was officially included in the SDR currency basket, which greatly increased the external attention paid toward RMB. The number of RMB investors in the foreign exchange market increased, and the use of RMB in cross-border trade also increased accordingly. The status of RMB in international settlement is becoming more and more important, and the exchange rate fluctuation of RMB to foreign currency has become a focus of investors' attention.

The internationalization of the RMB means that the offshore RMB exchange rate is largely determined by the market, but the offshore exchange rate risk will aggravate the volatility of the onshore exchange rate, severely disrupt China's financial market, and even affect the profits of Chinese foreign trade companies. Therefore, the effective prediction of and research into the RMB exchange rate will not only help the country and foreign trade companies to strengthen management and avoid foreign exchange risks but will also be a powerful supplement to the foreign exchange market theory.

Research regarding the prediction of the RMB exchange rate will mean trends of exchange rates can be detected earlier and necessary measures regarding the impact on future cash flow can be taken in advance to effectively avoid exchange rate risks. From a long-term perspective, this will help the central bank to further improve the RMB exchange rate market-oriented formation mechanism, allow the market to determine the exchange rate, increase the flexibility of the RMB exchange rate in two directions, and ensure the basic stability of the RMB exchange rate, so as to ensure that the exchange rate policy can promote economic development.

As an important macroeconomic variable, exchange rates have crucial impacts on macroeconomic operation under open conditions and the allocation of resources at the microeconomic level. Accurate forecasts of exchange rates are of great significance for future economic development and national investment decisions and also make important contributions to the economic development of the specific country and the world.

\section{Conclusions}

The currency market is one of the most efficient and complex markets, which makes it difficult to forecast future exchange rates. Some studies have developed several forecasting models to predict exchange rates. However, most of the previously developed methods fail to consider the ambiguities and uncertainties that time series present. In this paper, a novel 
fuzzy time series forecasting system based on a combined fuzzification strategy and the advanced optimization algorithm was proposed for use in USD/RMB and EURO/RMB exchange rate forecasting. The comparison experiments indicated that the proposed forecasting system is superior to some traditional models and the statistical models display better performance than artificial intelligence models. In addition, the statistical tests verified that the proposed forecasting system has significant differences from the compared models. Thus, the proposed forecasting system can provide a reference and basis for market management and investment.

Author Contributions: Conceptualization, J.Y. and M.T.; data curation, H.Z.; formal analysis, M.T.; investigation, I.A.; methodology, M.T.; supervision, X.D.; visualization, A.Z.; writing—original draft, J.Y.; writing-review and editing, M.T. and N.A. All authors have read and agreed to the published version of the manuscript.

Funding: This research was supported by Hubei Provincial Natural Science Foundation of China (No. 2021CFB034) and funding for post-doctoral work by the Department of Human Resources and Social Security of Hubei Province. The State Key Program of National Natural Science of China [No. 51239004] and the National Natural Science Foundation of China [No. 51309105].

Data Availability Statement: The data used to support the findings of this study are available from the corresponding author upon request.

Acknowledgments: The authors Jie Yin, He Zhang, and Aqeela Zahra have contributed equally to this paper. The authors would also like to offer deep thanks to Muhammad Tayyab and Xiaohua Dong from for their assistance in the successful completion of the work.

Conflicts of Interest: The authors declare that there are no possible conflict of interest regarding the publication of this paper.

\section{References}

1. He, K.; Chen, Y.; Tso, G.K.F. Forecasting exchange rate using Variational Mode Decomposition and entropy theory. Phys. A Stat. Mech. Appl. 2018, 510, 15-25. [CrossRef]

2. Kearney, F.; Cummins, M.; Murphy, F. Using extracted forward rate term structure information to forecast foreign exchange rates. J. Empir. Financ. 2019, 53, 1-14. [CrossRef]

3. Henríquez, J.; Kristjanpoller, W. A combined Independent Component Analysis-Neural Network model for forecasting exchange rate variation. Appl. Soft Comput. 2019, 83, 105654. [CrossRef]

4. Beckmann, J.; Czudaj, R. The impact of uncertainty on professional exchange rate forecasts. J. Int. Money Financ. 2017, 73, 296-316. [CrossRef]

5. Ponomareva, N.; Sheen, J.; Wang, B.Z. Forecasting exchange rates using principal components. J. Int. Financ. Mark. Inst. Money 2019, 63, 101131. [CrossRef]

6. Colombo, E.; Pelagatti, M. Statistical learning and exchange rate forecasting. Int. J. Forecast. 2020, 36, 1260-1289. [CrossRef]

7. Semiromi, H.N.; Lessmann, S.; Peters, W. News will tell: Forecasting foreign exchange rates based on news story events in the economy calendar. N. Am. J. Econ. Financ. 2020, 52, 101181. [CrossRef]

8. Wei, Y.; Sun, S.; Ma, J.; Wang, S.; Lai, K.K. A decomposition clustering ensemble learning approach for forecasting foreign exchange rates. J. Manag. Sci. Eng. 2019, 4, 45-54. [CrossRef]

9. Amat, C.; Michalski, T.; Stoltz, G. Fundamentals and exchange rate forecastability with simple machine learning methods. J. Int. Money Financ. 2018, 88, 1-24. [CrossRef]

10. Baffour, A.A.; Feng, J.; Taylor, E.K. A hybrid artificial neural network-GJR modeling approach to forecasting currency exchange rate volatility. Neurocomputing 2019, 365, 285-301. [CrossRef]

11. Balcilar, M.; Bouri, E.; Gupta, R.; Roubaud, D. Can volume predict Bitcoin returns and volatility? A quan-tiles-based approach. Econ. Model. 2017, 64, 74-81.

12. Bayer, S. Combining Value-at-Risk forecasts using penalized quantile regressions. Econ. Stat. 2018, 8, 56-77. [CrossRef]

13. Iqbal, M.; Naveed, A. Forecasting Inflation: Autoregressive Integrated Moving Average Model. Eur. Sci. J. ESJ 2016, 12, 83-92. [CrossRef]

14. Zhang, G.; Zhang, X.; Feng, H. Forecasting financial time series using a methodology based on autoregressive integrated moving average and Taylor expansion. Expert Syst. 2016, 33, 501-516. [CrossRef]

15. You, Y.; Liu, X. Forecasting short-run exchange rate volatility with monetary fundamentals: A GARCH-MIDAS approach. J. Bank. Finance 2020, 116, 105849. [CrossRef]

16. Wang, J.-Z.; Wang, J.-J.; Zhang, Z.-G.; Guo, S.-P. Forecasting stock indices with back propagation neural network. Expert Syst. Appl. 2011, 38, 14346-14355. [CrossRef] 
17. Ticknor, J.L. A Bayesian regularized artificial neural network for stock market forecasting. Expert Syst. Appl. 2013, 40, 5501-5506. [CrossRef]

18. He, K.; Ji, L.; Tso, K.F.G.; Zhu, B.; Zou, Y. Forecasting Exchange Rate Value at Risk using Deep Belief Network Ensemble based Approach. Procedia Comput. Sci. 2018, 139, 25-32. [CrossRef]

19. Zhou, Z.; Lin, L.; Li, S. International stock market contagion: A CEEMDAN wavelet analysis. Econ. Model. 2018, 72, 333-352. [CrossRef]

20. Zadeh, L.A. Fuzzy sets. Inf. Control. 1965, 8, 338-353. [CrossRef]

21. Song, Q.; Chissom, B.S. Fuzzy Time Series and its Models. Fuzzy Sets Syst. 1993, 54, 269-277. [CrossRef]

22. Torra, V. Hesitantfuzzy sets. Int. J. Intell. Syst. 2010, 25, 529-539. [CrossRef]

23. Jiang, P.; Yang, H.; Li, R.; Li, C. Inbound tourism demand forecasting framework based on fuzzy time series and advanced optimization algorithm. Appl. Soft Comput. 2020, 92, 106320. [CrossRef]

24. Bisht, K.; Kumar, S. Fuzzy time series forecasting method based on hesitant fuzzy sets. Expert Syst. Appl. 2016, 64, 557-568. [CrossRef]

25. Yang, H.; Jiang, Z.; Lu, H. A Hybrid Wind Speed Forecasting System Based on a 'Decomposition and Ensemble' Strategy and Fuzzy Time Series. Energies 2017, 10, 1422. [CrossRef]

26. Dong, H.; Li, T.; Ding, R.; Sun, J. A novel hybrid genetic algorithm with granular information for feature selection and optimization. Appl. Soft Comput. 2018, 65, 33-46. [CrossRef]

27. Ding, Y.; Fu, X. Kernel-based fuzzy c-means clustering algorithm based on genetic algorithm. Neurocomputing 2016, 188, 233-238. [CrossRef]

28. Abedinpourshotorban, H.; Shamsuddin, S.M.; Beheshti, Z.; Jawawi, D.N.A. Electromagnetic field optimization: A physicsinspired metaheuristic optimization algorithm. Swarm Evol. Comput. 2016, 26, 8-22. [CrossRef]

29. Bouchekara, H. Solution of the optimal power flow problem considering security constraints using an improved chaotic electromagnetic field optimization algorithm. Neural Comput. Appl. 2019, 32, 2683-2703. [CrossRef]

30. Li, H.; Wang, J.; Li, R.; Lu, H.Y. Novel analysis-forecast system based on multi-objective optimization for air quality index. J. Clean. Prod. 2019, 208, 1365-1383. [CrossRef]

31. Wang, J.; Du, P.; Hao, Y.; Ma, X.; Niu, T.; Yang, W. An innovative hybrid model based on outlier detection and correction algorithm and heuristic intelligent optimization algorithm for daily air quality index forecasting. J. Environ. Manag. 2020, 255 , 109855. [CrossRef] 